\title{
Evaluation of a simple anti-sway control without start-up delay and over-travel
}

\author{
Akio YAMAMOTO* and Hiroki WATANABE*,** \\ * Department of Precision Engineering, The University of Tokyo \\ 7-3-1 Hongo, Bunkyo-ku, Tokyo, 113-8656, Japan \\ E-mail: akio@aml.t.u-tokyo.ac.jp \\ ** Hitachi Power Solutions Co.,Ltd. \\ Hitachi-shi, Ibaraki, Japan
}

Received: 1 April 2019; Revised: 5 August 2019; Accepted: 9 September 2019

\begin{abstract}
This paper evaluates a simple anti-sway control algorithm for fixed-speed cranes. Anti-sway control of suspended loads under cranes has been widely studied, and input shapers have been found as powerful tools. Whereas typical input shapers require variable-speed control for cranes, unity-magnitude (UM) shaper can be applied to fixed-speed cranes. However, UM shaper introduces a start-up delay and over-travel, and thus might not be user-friendly if applied to human-operated cranes. In this work, much simpler anti-sway algorithm for fixed-speed cranes with non-damped oscillations is implemented and evaluated in terms of its robustness. The algorithm is based on the conventional zero-vibration $(\mathrm{ZV})$ shaper, and consists of two sub methods. In the first sub method, the command from an operator is subdivided into a pulse train, which is then fed to $\mathrm{ZV}$ shaper. Resulting shaped command is a sum of the original command and one short pulse to suppress the residual oscillation, which is called suppression pulse. Although the command cannot suppress sway during transportation, the crane operated based on the command does not cause start-up delay. On the other hand, the command creates over-travel due to the suppression pulse. To eliminate the over-travel, the second sub method adds a rewind pulse, which is also fed to ZV shaper before being added. The resulting total command exhibits no start-up delay and no over-travel. Experiments using a miniature experimental crane verified the suppression of the residual oscillation, as well as no start-up delay and no over-travel. The paper analyzes its sensitivity against the modeling error. The analyses show that the method is more sensitive to the modeling error when the operation command has a longer duration. Therefore, the algorithm would be effective for cranes with a short-traveling time and/or a long oscillation period.
\end{abstract}

Keywords : Input shaping, ZV shaper, Anti-sway, Oscillation suppression, Crane, ON/OFF control, Time-delay

\section{Introduction}

Cranes, such as gantry cranes, are widely used in factories, warehouses, or many other industrial facilities. Typical cranes lift and transport loads using hoist ropes, which are flexible and can easily sway. When a load is transported using a crane, the flexibility of the rope leads to heavy sway of the transported load. Such a heavy sway disturbs quick operation and precise positioning. Moreover, the sway, especially the resulting residual oscillation after the trolley has stopped, is hazardous to the crane operator or the facility itself. Therefore, a huge number of studies have been done to prevent the sway and the residual oscillation.

Generally, there are two approaches to prevent the sway/oscillation: feedback control and feed-forward control. While feedback control shows robust performance compared to feed-forward approach (Alhassan, et al. 2015), it requires additional sensors installed on the cranes, although there are some studies that virtually realize feedback control without sensors, by using simulation (Wada, et al. 2017). On the other hand, feed-forward control can be easily implemented without modifications to the cranes, and thus preferred in many applications. In some studies, both of them are combined to achieve high performance and robustness (Miyata, et al. 2001; Sorensen, et al. 2005). 

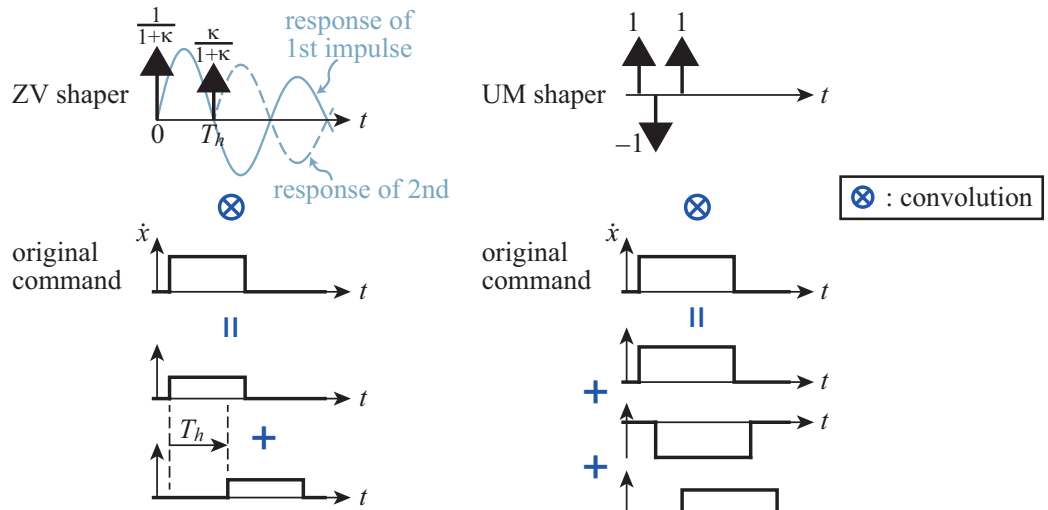

II

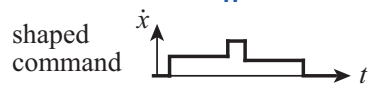

(a) ZV shaper

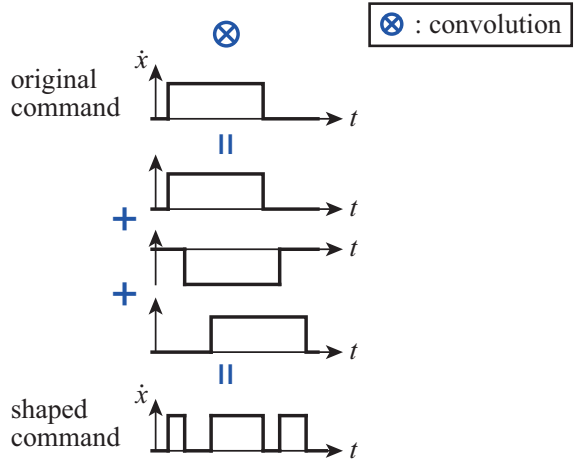

(b) UM shaper

Fig. 1 ZV shaper (a) and UM shaper (b). In these input shapers, impulses are convolved with an original operation command to provide a shaped command that does not excite oscillations of the load. When a simple ON$\mathrm{OFF}$ command is given as the original command, $\mathrm{ZV}$ shaper creates variable-speed command. On the other hand, UV shaper creates ON-OFF type command that can be fed to a fixed-speed crane.

One of the most preferred feed-forward approaches would be input shaping (Singer 1988; Singer and Seering 1990; Singhose, et al. 1996; Pao and Singhose 1996). In input shaping, an original command is filtered by an input-shaper, which is a kind of FIR filter. In the past studies, many different input shapers have been proposed as reviewed in (Singhose 2009). The most basic one among them would be zero-vibration (ZV) shaper, based on posicast control done by Smith (Smith 1957). ZV shaper consists of two-impulses, whose time-interval is equal to a half of the oscillation period of the target system. The amplitude ratio of the two impulses matches the ratio of the absolute peak values of two adjacent half-cycles of the oscillation, as shown in Fig. 1(a). As the responses of the two impulses cancel each other, convolution of this ZV shaper with any motion command results in a shaped command that does not excite oscillations. Based on ZV shaper, many variants have been developed to enhance robustness, such as ZVD (zero vibration and derivative) shaper, EI (extra insensitivity) shaper, and SI (specified-insensitivity) shaper (Singhose, et al. 1990; Singhose, et al. 1996).

These input shapers basically shift and add the original operation command as shown in Fig. 1(a). The resulting command shape is typically complicated, even if the original command is a simple ON/OFF command. Therefore, to apply these input shapers to actual cranes, the cranes must have a capability to respond to variable-speed commands. However, typical industrial cranes can only move at one or two fixed speeds. Therefore, it is not possible to apply ZV shaper and their variants to those industrial cranes.

This problem can be solved by using UM (unity-magnitude) shaper (Pao and Singhose 1996; Gürleyük 2007). The shaper, in its most basic type, consists of three impulses with the same amplitude, but different in polarities. If an ON/OFF command is fed to UM shaper, the resulting signal becomes an ON/OFF pulse train, as illustrated in Fig. 1(b). Such ON/OFF pulse trains can be directly fed to fixed-speed cranes (Sorensen, et al. 2005). However, UM shaper introduces a delay in crane motion. Therefore, when applied to a human-operated crane, the motion created by UM shaper would not be felt natural for crane operators. Regarding start-up, for example, the motor is activated for a short period by the first pulse, then cut off for a while before being activated again. The resulting discontinuous behavior would be felt unnatural. In addition to the start-up issue, the delay involved in the input shaper causes another problem, which is over-travel. As the shaped command lags the original command, the crane will keep moving, even after the original command ends. Therefore, when operated by a human operator, he/she needs to switch off the operation button a short time before the trolley reaches the target position, by estimating the length of the over-travel. This makes precise positioning difficult.

The delay issue is not unique to UM shaper, but a universal issue of input shapers. Therefore, it has been discussed in many studies (Singer, et al. 1997; Cutforth and Pao 2003; Zhao, et al. 2016; Zhao and Tomizuka 2017; Yaovaja, et al. 2017). Some studies have discussed the delay issue focusing on the over-travel problem (Sorensen, et al. 2005; Vaughan and Singhose 2009; Vaughan, et al. 2011). However, they mostly require variable-speed control or need to combine feedback control that requires modification to the crane system. There seems no clear solution to handle the start-up delay and over-travel problems, for unmodified fixed-speed cranes. 
The input shapers typically deal with damped oscillations (non-damped oscillations are dealt with as a special case of damped oscillations). In many practical cranes, however, suspended loads can often be regarded as non-damped (or hardly-damped) oscillators, which are theoretically much simpler than damped oscillations. Therefore, if we limit our focus to non-damped oscillations, anti-sway control can be implemented in a much simpler way. In this paper, targeting at non-damped fixed-speed cranes, we implement and evaluate a much simpler algorithm for reducing the residual oscillation.

The simple algorithm described in the paper exploits the nature of ZV shaper and thus can suppress the residual oscillation. The algorithm involves two sub methods; one is pulse subdivision and the other is rewind pulse. Pulse subdivision creates a command without start-up delay, and rewind pulse compensates the over-travel. In both sub methods, $\mathrm{ZV}$-shaper is utilized to suppress the oscillation. The combined algorithm can prevent start-up delay and over-travel in a quite simple way. On the other hand, unlike the input shapers, the algorithm does not suppress the oscillation during transportation, which could be a drawback.

The crane motions realized by these methods resemble the motions created by experienced crane operators. In factories, experienced operators often add some short pulse commands to suppress a residual oscillation and/or overtravel. The method described here automatically creates those short pulse commands. In that sense, this work provides a theoretical explanation to the operations of those experienced operators.

In the following, the paper describes the concept of the implemented method in sections 2 and 3. Section 2 discusses the concept and formulation of pulse subdivision, whereas section 3 explains rewind pulse. In section 4, the method is applied to a miniature crane to verify its effectiveness. The experimental results confirm that the implemented method can transport a load without start-up delay, residual oscillations, and over-travel. In section 5, the method is analyzed in terms of its robustness to show the disadvantage, which comes as a trade-off for the simplicity of the method. Section 6 is the conclusions.

\section{Pulse subdivision: zero start-up delay anti-sway control}

This work assumes a manually operated fixed-speed crane with non-damped (or hardly-damped) oscillations of suspended loads. Here, 'manually operated' means that the original operation command is given by a human operator using a control pendant, in a simple ON/OFF manner. The anti-sway algorithm, evaluated in this work, takes the original operation command and generates a shaped command that is fed to the motor of the crane. The algorithm is divided into two sub methods, and the first one, pulse subdivision, is described in this section.

\subsection{Concept}

If an ON/OFF operation command from a control pendant is directly fed to a $\mathrm{ZV}$ shaper, the resulting shaped command will have multiple speeds as illustrated in Fig. 1(a). Such a command cannot be accepted by fixed-speed cranes. To generate a single-speed command, this work first subdivides the original command into a pulse train, which is shown in Fig. 2 as 'synchronized pulse train'. As the original operation command (a-1) becomes ON, an oscillator starts generating pulses (a-2). Their pulse-duration is same as a half of the oscillation period of the suspended load, which is denoted as $T_{h}$. The pulse generation will continue until the operation command turns off. The generated pulse train is, then, fed to ZV shaper (a-3) to create a shaped command (a-4). The used ZV shaper has two impulses with a time interval of $T_{h}$ and with the same height, as we assume non-damped oscillations. The resulting shaped command becomes a combination of the original command and one short additional pulse, as illustrated in the figure. This additional pulse is referred to as suppression pulse. The process, in which an original input command is replaced by a pulse train and then fed to ZV shaper, is referred to as pulse subdivision. When this shaped command is given to a crane, the crane will move in the same trajectory as with the original command, except for the final small motion caused by the suppression pulse. As the crane motion during the operation switch is being ON is exactly the same as the manual operation, an operator can use the system in the same manner as typical cranes.

The behavior of pulse subdivision depends on the duration of the original command. The above explained is for the case when the operation switch is turned off during a former half of an oscillation cycle (in other words, during the pulse train is ON). This case is referred to as case I. If the switch is turned off during a latter half of an oscillation cycle as in Fig. 2(b-1), the shaped signal will look slightly different as in Fig. 2(b). This case is referred to as case II. In this case, if the pulse train is simply fed to $\mathrm{ZV}$ shaper, the resulting command will be a single pulse that has a longer duration than the original command, as in (b-3). In this case, the crane keeps moving even after the operation switch is turned off which 

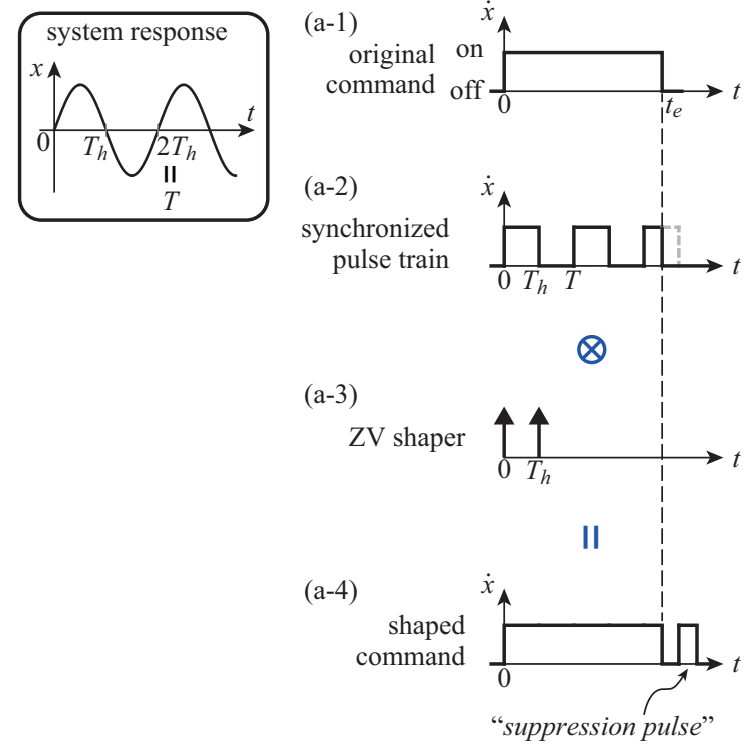

(a) case I

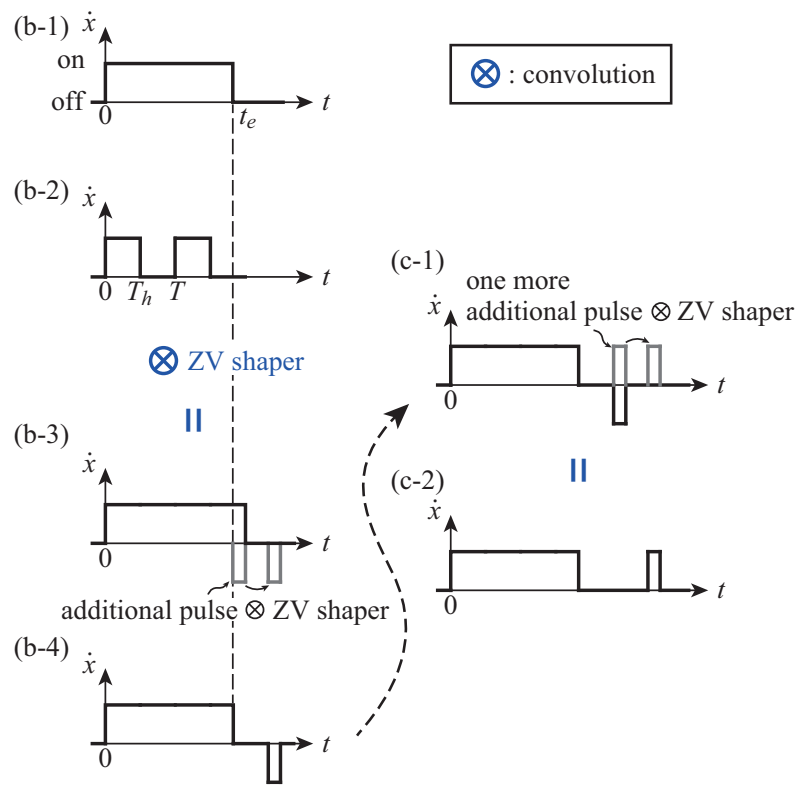

(b) case II (c) case II'

Fig. 2 Basic concept of pulse subdivision. A given original operation command is first converted into a pulse train, which is then convolved with ZV shaper. Resulting command signal is a sum of the original operation command and one suppression pulse. The polarity of the suppression pulse depends on the length of the original command.

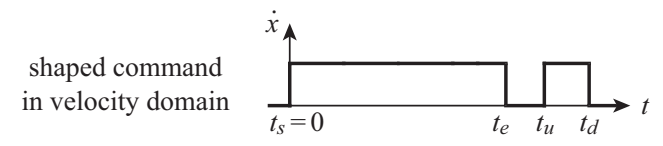

II

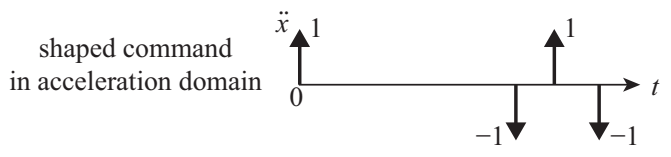

Fig. 3 Representation of pulse subdivision using acceleration impulses

can deteriorate usability. Therefore, we cancel the exceeding part of the signal by adding a negative pulse, as illustrated in (b-3). The negative pulse is also fed to ZV shaper to be replicated. The resulting shaped command (b-4) will be, again, a combination of the original input and a suppression pulse; but this time its polarity is negative. If a positive suppression pulse is preferred, we can add a positive pulse again, to cancel the negative suppression pulse, as shown in Fig. 2(c-1). The added positive pulse is also fed to $\mathrm{ZV}$ shaper to be replicated, which appears as a positive suppression pulse, as in (c-2). As a special case, if the operation switch is turned off just at an even multiple of $T_{h}$, there will be no additional pulse, since the original command does not create any residual oscillations.

\subsection{Formulation}

In an actual implementation, we do not really follow the above process, such like making a pulse train, and feeding it to ZV shaper. Instead, we can simply let the original command pass through, and after the original command is switched off, we can calculate and add a suppression pulse. Therefore, what actually matters is the timings of the suppression pulse.

From the concept described above, we can easily derive the timing of the suppression pulse, in a graphical way. Here, however, we derive them mathematically for better understanding of the method. The derivation is based on the work by Singer (Singer 1988). Here we denote the start and the end of the original and suppression pulses as $t_{s}, t_{e}, t_{u}$, and $t_{d}$, as illustrated in Fig. 3. The variables $t_{s}=0$ and $t_{e}$ represent the start and the end of the original command, and $t_{u}$ and $t_{d}$ are the moments of the rising (up) and falling (down) of the suppression pulse. For case I, $t_{u}$ is smaller than $t_{d}\left(t_{u}<t_{d}\right)$, but for case II, $t_{d}$ is smaller $\left(t_{d}<t_{u}\right)$. In the acceleration domain, the shaped signal can be represented using four impulses at those four moments. Therefore, the response of the system can be calculated by combining the four impulse responses. 
Here we assume undamped 2nd-order oscillation system with a transfer function $H(s)=\omega_{n} /\left(s^{2}+\omega_{n}^{2}\right)$. Its impulse response is obtained by inverse Laplace transform as $h(t)=\sin \left(\omega_{n} t\right)$. The sum of responses of the four impulses, $h_{\text {all }}(t)$, is

$$
\begin{gathered}
h_{\text {all }}(t)=\sin \left(\omega_{n} t\right)-\sin \left(\omega_{n}\left(t-t_{e}\right)\right)+\sin \left(\omega_{n}\left(t-t_{u}\right)\right)-\sin \left(\omega_{n}\left(t-t_{d}\right)\right) \\
=\sin \left(\omega_{n} t\right)\left\{1-\cos \left(\omega_{n} t_{e}\right)+\cos \left(\omega_{n} t_{u}\right)-\cos \left(\omega_{n} t_{d}\right)\right\} \\
\quad+\cos \left(\omega_{n} t\right)\left\{\sin \left(\omega_{n} t_{e}\right)-\sin \left(\omega_{n} t_{u}\right)+\sin \left(\omega_{n} t_{d}\right)\right\}
\end{gathered}
$$

To suppress the residual oscillation, $h_{\text {all }}(t)=0$ must be satisfied. Therefore, we obtain

$$
\left\{\begin{array}{l}
1-\cos \left(\omega_{n} t_{e}\right)+\cos \left(\omega_{n} t_{u}\right)-\cos \left(\omega_{n} t_{d}\right)=0 \\
\sin \left(\omega_{n} t_{e}\right)-\sin \left(\omega_{n} t_{u}\right)+\sin \left(\omega_{n} t_{d}\right)=0
\end{array}\right.
$$

Rewriting this as

$$
\begin{cases}1-\cos \left(\omega_{n} t_{e}\right)=\cos \left(\omega_{n} t_{d}\right)-\cos \left(\omega_{n} t_{u}\right) & \ldots(*) \\ \sin \left(\omega_{n} t_{e}\right)=\sin \left(\omega_{n} t_{u}\right)-\sin \left(\omega_{n} t_{d}\right) & \ldots(* *)\end{cases}
$$

and calculating $(*)^{2}+(* *)^{2}$, we obtain

$$
\cos \left(\omega_{n} t_{e}\right)=\cos \left(\omega_{n}\left(t_{d}-t_{u}\right)\right)=\cos \left(\omega_{n} t_{d}\right) \cos \left(\omega_{n} t_{u}\right)+\sin \left(\omega_{n} t_{d}\right) \sin \left(\omega_{n} t_{u}\right)
$$

From $(*)^{2}-(* *)^{2}$,

$$
\begin{aligned}
2 \cos \left(\omega_{n} t_{e}\right)\left(\cos \left(\omega_{n} t_{e}\right)-1\right) & =\cos \left(2 \omega_{n} t_{d}\right)+\cos \left(2 \omega_{n} t_{u}\right)-2 \cos \left(\omega_{n}\left(t_{d}+t_{u}\right)\right) \\
& =2 \cos \left(\omega_{n}\left(t_{d}+t_{u}\right)\right) \cos \left(\omega_{n}\left(t_{d}-t_{u}\right)\right)-2 \cos \left(\omega_{n}\left(t_{d}+t_{u}\right)\right) \\
& =2 \cos \left(\omega_{n}\left(t_{d}+t_{u}\right)\right)\left\{\cos \left(\omega_{n}\left(t_{d}-t_{u}\right)\right)-1\right\}
\end{aligned}
$$

Substituting the relation in Eq. (4) gives

$$
\cos \left(\omega_{n} t_{e}\right)=\cos \left(\omega_{n}\left(t_{d}+t_{u}\right)\right)=\cos \left(\omega_{n} t_{d}\right) \cos \left(\omega_{n} t_{u}\right)-\sin \left(\omega_{n} t_{d}\right) \sin \left(\omega_{n} t_{u}\right)
$$

Therefore, from Eqs. (4) and (6), we have

$$
\sin \left(\omega_{n} t_{u}\right) \sin \left(\omega_{n} t_{d}\right)=0
$$

If we simply set $t_{u}=T_{h} n$, such that $\sin \left(\omega_{n} t_{u}\right)=0$ and $\cos \left(\omega_{n} t_{u}\right)=-1$, the smallest $t_{d}$ to satisfy $t_{d}>t_{e}$ is obtained as $t_{e}+T_{h}$ from Eq. (2). Here, $T_{h}$ is equal to $\pi / \omega_{n}$ and $n$ is the smallest odd integer that satisfies $T_{h} n>t_{e}$. This solution holds for both of the two cases: $t_{u}<t_{d}$ for case I and $t_{u}>t_{d}$ for case II.

To summarize, pulse subdivision is a method to add a suppression pulse to the original operation pulse, and the rising and falling timings of the suppression pulse are defined as follows.

$$
\left[t_{u}, t_{d}\right]=\left[T_{h} n, t_{e}+T_{h}\right] \quad\left(n=\operatorname{ceil}\left(t_{e} / T+0.5\right) \times 2-1\right)
$$

In case II, $t_{d}$ is smaller than $t_{u}$, which means the suppression pulse is a negative pulse.

It should be noted that although the pulse subdivision algorithm is based on ZV-shaper in its concept, the algorithm cannot be expressed in a form of FIR filter. However, as we can easily obtain the timing of the suppression pulse as explained above, its implementation is considerably simple.

\section{Rewind pulse: over-travel compensation}

In the above pulse subdivision, a suppression pulse is added after the original command. Therefore, the final position of the crane is different from where the original command was turned off. If the suppression pulse is positive, which is case I, the crane will over-travel. For case II, where the suppression pulse has negative polarity, the crane will move back; in other words, it under-travels. To settle the load at the position where the switch on the control pendant is turned off, the second method, rewind pulse, is described in this section.

The amount of over-travel or under-travel is proportional to the integral of the signal after the switch is turned off. To make the integral zero, this work adds another pulse with the opposite polarity to the suppression pulse. For example, for a positive suppression pulse, a negative pulse with its half width is added to cancel out a half of the suppression pulse. The newly added pulse is also fed to ZV shaper, which replicates the pulse. The replication makes the total area of the 


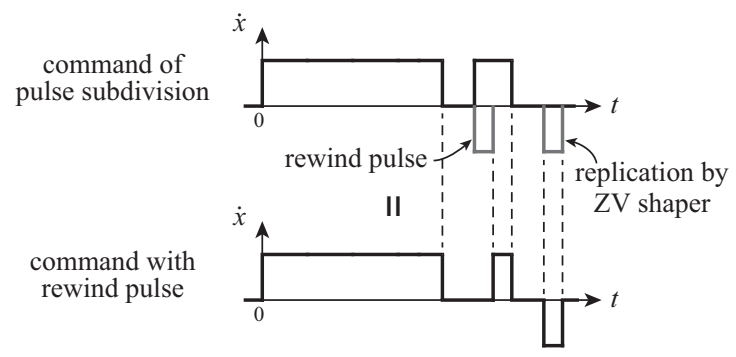

Fig. 4 Rewind pulse. To settle the transported load at the target position, a short rewind pulse is added to the suppression pulse. The rewind pulse has a half time-length of the suppression pulse. As the rewind pulse is convolved with ZV shaper to be replicated, the total time-length becomes equal to the suppression pulse and over/under-travel can be eliminated.

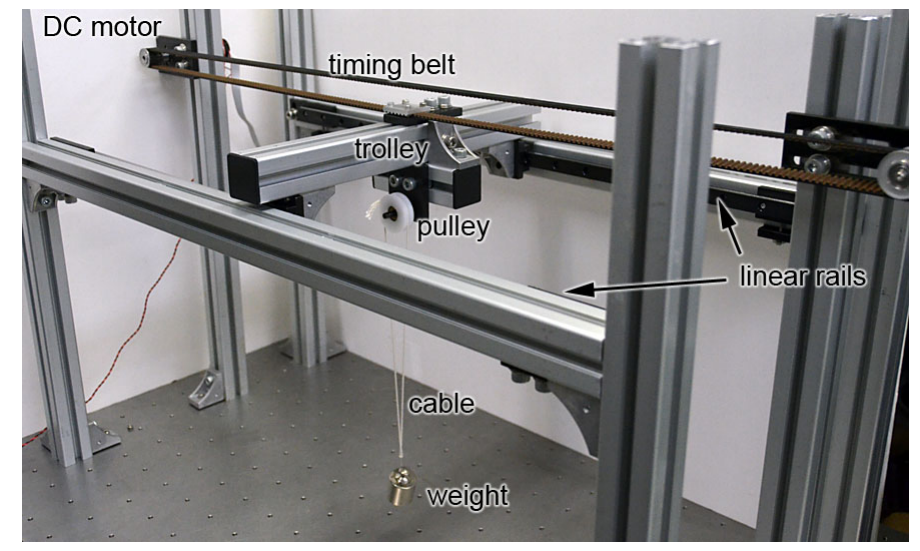

Fig. 5 Experimental setup. A miniature crane system was built for the experiments. The DC motor was controlled by a feedback velocity controller to emulate fixed-speed motions. The trolley was moved by the DC motor through the timing belt. A weight was hung below the trolley using a pulley and a cable. The pulley was supported by a ball bearing to minimize the damping of the oscillation.

negative pulses being equal to the positive suppression pulse. This makes the integral equal to zero, which means there is no over-travel. Resulting time chart is shown in Fig. 4.

The timing to add the rewind pulse is arbitrary. In this work, we assume that the smaller number of resultant pulses would be felt more natural, and thus propose to add the rewind pulse such that it coincides with the former half of the suppression pulse. By doing so, the total number of pulses becomes three, including the first original command.

\section{Experiments}

\subsection{Setup}

To confirm the validity of the above algorithm, an experimental miniature crane system was built as shown in Fig. 5 . The trolley was arranged on linear rails and was driven by a DC-motor through a timing belt. The DC motor was equipped with an encoder to facilitate feedback velocity control. A fixed-speed proportional feedback controller was implemented on a digital signal processing (DSP) board (D-space, DS1104). The anti-sway algorithm was also implemented on the same DSP system.

Beneath the trolley, a cable was connected using a pulley and a ball bearing to minimize the damping of the cable oscillation. A weight of $0.2 \mathrm{~kg}$ was attached to the lower end of the cable, emulating a load. The oscillation period was measured as 1.146 seconds, which was used as a parameter of the anti-sway algorithm. The measured ratio of the absolute peak values of two adjacent half-cycles of the oscillation was 0.996 , which shows that there is almost no damping (the corresponding damping ratio $\zeta$ is $1.28 \times 10^{-3}$ ). In the experiments, the motion of the trolley and the load were measured by a high-speed camera with an offline motion tracking function (KEYENCE, VW-6000).

\subsection{Results}

Figure 6 compares motions of the load (weight) for three different commands: original operation command, with pulse subdivision, and with pulse subdivision plus rewind pulse. The upper three plots (a-c) show the commands fed to the crane. The two shaped commands (b, c) are both based on the same operation command (a), which was created on the 
(a) $\begin{aligned} & \text { operation command } \\ & \text { (without anti-sway) }\end{aligned}$

(b)

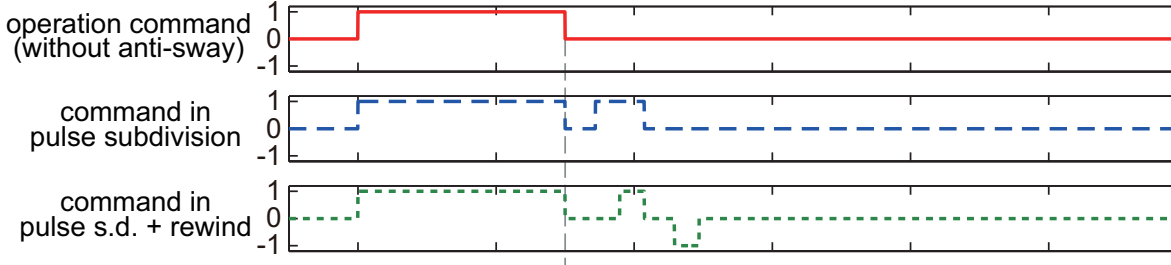

(d)

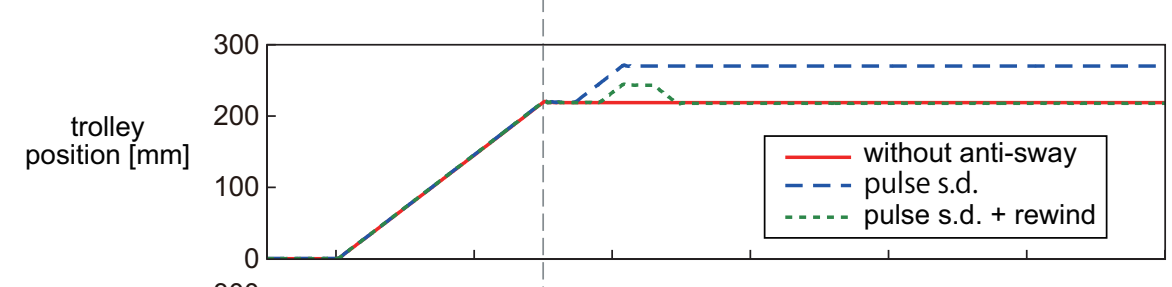

(e)

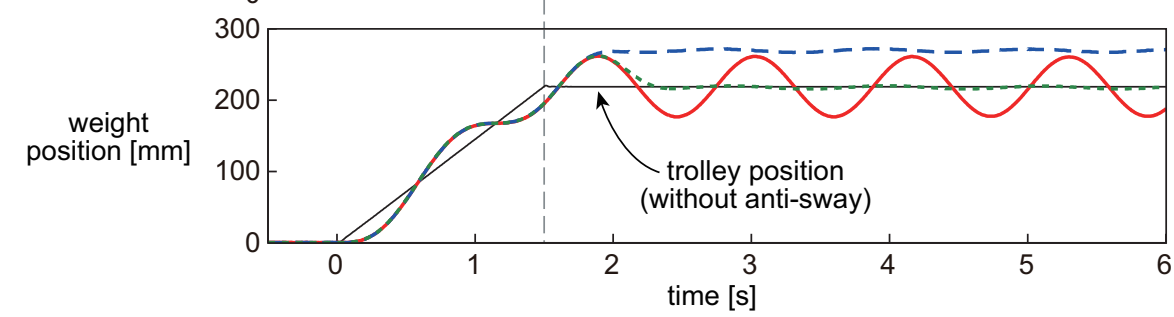

Fig. 6 Experimental results for original operation command, with pulse subdivision, and with pulse subdivision plus rewind pulse. The plots in (d) show that the trolley motions were the same during the operation command is $\mathrm{ON}$, for all the three conditions. When pulse subdivision is applied, the trolley moved additionally to suppress the residual oscillation of the weight. When rewind pulse was further applied, the trolley and the weight settled at the target location.

same DSP system to emulate operator's switch operation. The lower two plots $(\mathrm{d}, \mathrm{e})$ compare the motions of the trolley and the weight in the three cases. Trolley position for the original operation command is also plotted in the bottom plot (e), as a reference.

When the original command was directly given, the weight heavily oscillated after the trolley had stopped. On the other hand, when pulse subdivision was applied, the weight did not oscillate after the transportation, due to the suppression pulse. Here, it should be noted that the trolley (and the weight) motion was the same for both the original operation command and in pulse subdivision, during the operation command is ON. Therefore, an operator can easily predict the behavior of the crane, and can operate the system in the same manner as typical cranes. Actually, the motion realized by pulse subdivision is similar to the motions created by experienced crane operators for typical cranes; experienced operators often add a short pulse after the transportation to suppress the residual oscillation.

Although the pulse subdivision algorithm successfully suppressed the residual oscillation, it produced a certain amount of over-travel due to the suppression pulse. On the other hand, when the rewind pulse method is combined, the trolley and the weight settled at the location where the operation switch was turned off. When the operation command became zero (which means the operation switch was turned off), the trolley was at around $215 \mathrm{~mm}$ from the start point. Then, the final settling location of the weight was the same 215 -mm point, which means the over-travel was successfully compensated. Therefore, an operator can simply turn off the operation switch when the trolley has arrived at the desired location. These results clearly validate the effectiveness and usefulness of the pulse subdivision and rewind pulse algorithm.

\subsection{Comparison with UM shaper}

Next, the motion of the algorithm was compared with UM shaper, which can also be utilized for a fixed-speed crane. Figure 7 shows the motions with UM shaper, together with the motions of the proposed algorithm as a reference. When a simple ON/OFF operation command (a) is fed to UM shaper, the resulting command (b) becomes intermittent pulses in the beginning of the motion. As a result, the crane speed fluctuates intensely in the beginning as shown in (c); it starts moving, stops suddenly for a short moment, and then moves again. On the other hand, when the pulse subdivision and rewind pulse algorithm was applied, the crane smoothly moves, as in the normal operation.

Looking at the plot (d), it can be found that the motion of the weight settled down much faster with UM shaper than with the proposed algorithm. Also, UM shaper even prevents the sway motion during transportation. Regarding the 
(a) operation command $0_{-1}^{1}$

(b) shaped command ${ }_{\text {by UM shaper }}^{1}$

(c)

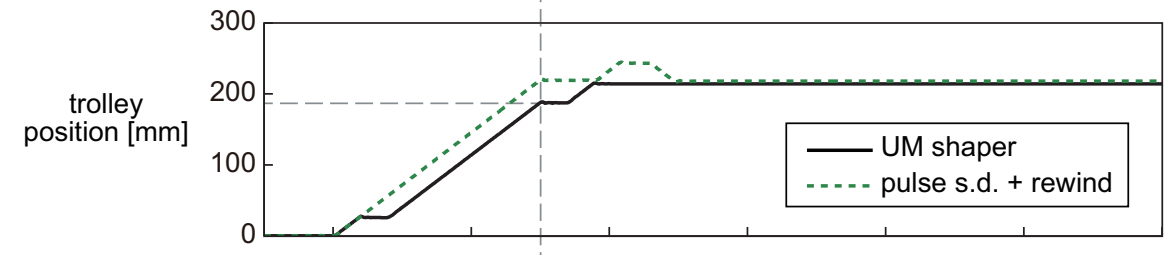

(d)

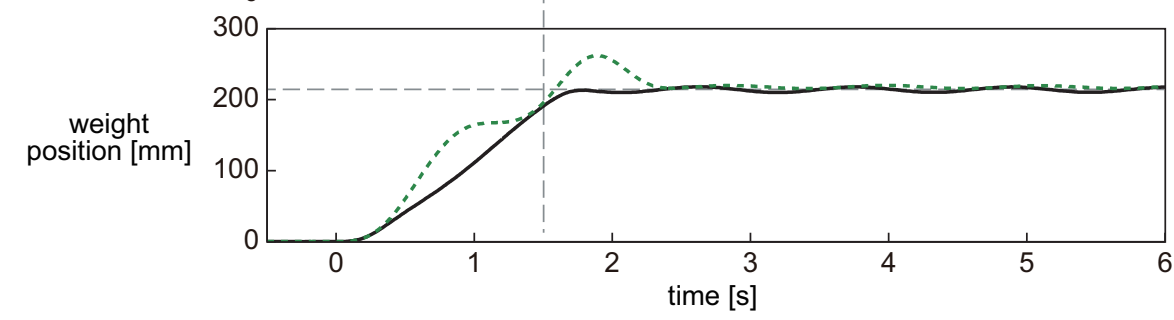

Fig. 7 Comparison with UM shaper. The motion of the proposed method (pulse subdivision and rewind pulse) is compared with that of UM shaper. Whereas UM shaper creates oscillation-free weight motion as shown in (d), its trolley motion is rather complicated compared to the straightforward motion of the proposed method. Also, the final settling position is different from the position where the original operation command was turned off.

settling location, it can be found that the final locations are the same for the two cases. Therefore, we could say, in one sense, that there is no over-travel in UM shaper. These comparisons highlight the advantages of UM shaper. But from the viewpoint of an operator, the story is different. When the operator turned off the switch at around 1.5 second, the trolley was at $187 \mathrm{~mm}$. However, the final location was at around $215 \mathrm{~mm}$, which means that the final location is different from where the operator intended. In that sense, we can say that the crane over-traveled from the intended location. In automated operation, this over-travel would not be a problem and UM shaper performs better than the proposed algorithm. However, for human operation, the proposed algorithm would be much more intuitive.

\section{Robustness analysis}

As described in section 2, the timings of the pulses in the proposed method are calculated based on the period of the oscillation, $T$. In the above experiments, the period was experimentally measured with enough precision, and was used in the algorithm. Such precise measurement, however, is not always possible in practice. In real cranes, we can only roughly estimate the period, possibly from the length of the cables. Therefore, it is quite important to investigate robustness of the algorithm against a modeling error of the oscillation period.

In actual systems, many other factors can affect the oscillation suppression performance. For example, frictions, fluctuation of the motor speed, and any other non-linear responses of the motor, may affect the performance. Theoretically, however, the only factor that can affect the suppression performance is the oscillation period $T$, and therefore, this section focuses on the modeling error of the oscillation period.

\subsection{Sensitivity of pulse subdivision}

First, the amplitude of the residual oscillation is calculated for pulse subdivision with a modeling error. Here we assume that the real oscillation period and its half are $T$ and $T_{h}=T / 2$, whereas the modeled ones are $\hat{T}$ and $\hat{T}_{h}=\hat{T} / 2$. Then, the directions $\left(A_{1}\right.$ to $\left.A_{4}\right)$ and timings $\left(t_{1}\right.$ to $\left.t_{4}\right)$ of the four acceleration impulses of the shaped command become

$$
\left\{\begin{array}{l}
{\left[A_{1}, A_{2}, A_{3}, A_{4}\right]=[1,-1,1,-1]} \\
{\left[t_{1}, t_{2}, t_{3}, t_{4}\right]=\left[0, t_{e}, \hat{T}_{h} n, t_{e}+\hat{T}_{h}\right] \quad\left(n=\operatorname{ceil}\left(t_{e} / \hat{T}+0.5\right) \times 2-1\right)}
\end{array}\right.
$$

Here, $t_{1}$ and $t_{2}$ represent the start and the end of the original command, and $t_{3}$ and $t_{4}$ are for the suppression pulse (variables are renamed from section 2 for simplicity). The forth impulse will appear earlier than the third one for case II, but the order of the appearance does not affect the calculation. Then, the normalized amplitude of the residual oscillation can be 


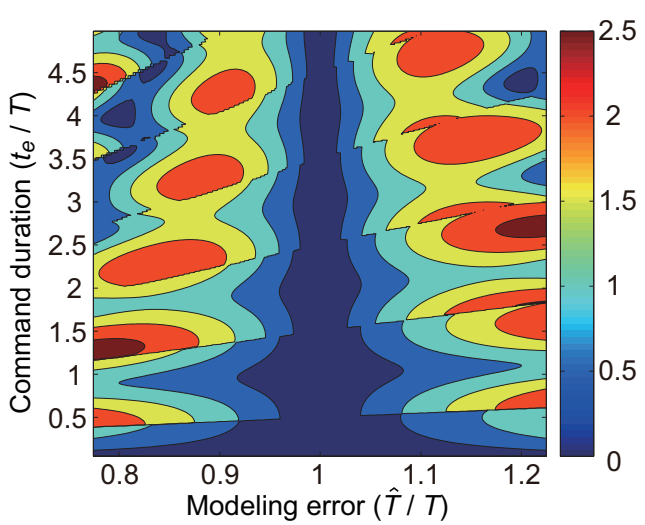

(a) pulse subdivision

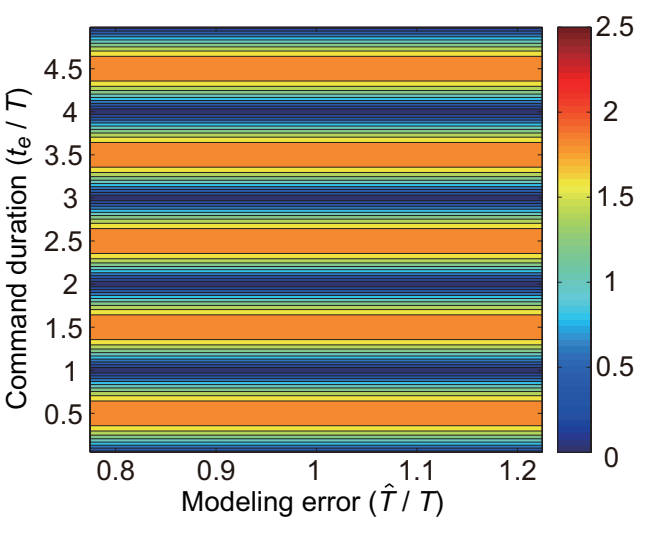

(b) normal operation

Fig. 8 Amplitudes of the residual oscillation for pulse subdivision (a) and normal operation (b). In pulse subdivision, estimation error of the oscillation period considerably affects the residual oscillation. Furthermore, as the duration of the command becomes longer, the method becomes more sensitive to the estimation error. In normal operation, the amplitude of the residual oscillation is simply determined by the phase of the oscillation when the operation command is turned off; it does not depend on the duration of the command.

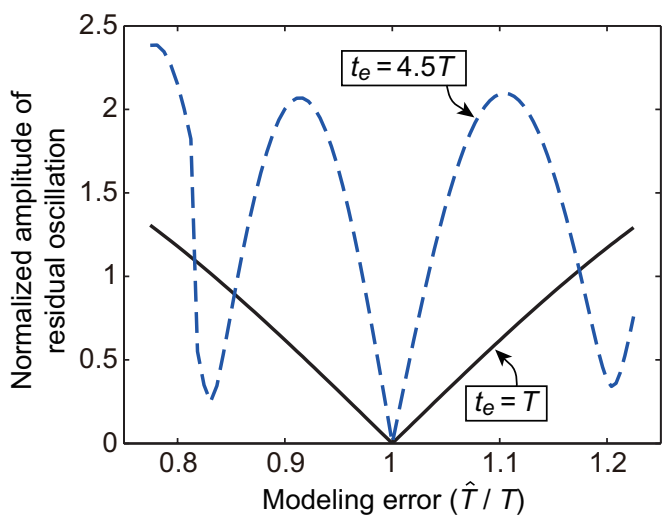

Fig. 9 Intersections of Fig. 8(a) at different command durations. The oscillation suppression performance becomes more sensitive to the modeling error, as the command duration becomes longer.

calculated as

$$
A_{r}=\left|\sum_{i=1}^{4} A_{i} e^{\omega_{n} t_{i}}\right|
$$

where $\omega_{n}$ is the angular frequency of the oscillation and is $\omega_{n}=2 \pi / T$.

Figure 8(a) shows the result. For a comparison, the residual oscillation amplitude of the original command is plotted in Fig. 8(b). As the operation based on the original command is not affected by the modeling error, the plot is uniform along the horizontal axis. The plot of Fig. 8(a) looks sectioned. This sectioning comes from the fact that $n$ in Eq. (9) is an integer. The section lines correspond to the odd numbers of $n(n=1,3,5, \ldots)$.

In pulse-subdivision, the modeling error accumulates as the length of the original input becomes larger. Therefore, for a longer pulse, which corresponds to larger $t_{e}$, the result becomes more sensitive to the modeling error. For a short pulse, such as $t_{e} \approx T$, the normalized amplitude is less than about 0.3 within $\pm 5 \%$ modeling error (see Fig. 9). However, for a longer pulse, such as $t_{e} \approx 4.5 T$, less than $\pm 1 \%$ error is allowed to achieve the same level of suppression. This characteristics are quite different from typical input shapers. In typical input shapers, the performance is generally independent from the command duration. Therefore, we can say that pulse subdivision is more effective for short time operation; in other words, it is useful for cranes with longer oscillation periods and/or shorter traveling time.

Figure 10 shows the ratio of the two plots in Fig. 8. The ratio being less than 1, which is indicated by red color, means that the pulse subdivision suppresses the oscillation compared to the normal operation. Blue color means the ratio exceeds 1 ; in other words, oscillations are enlarged. It can be found that the algorithm enlarges the residual oscillation in some situations. In most of those situations, the length of the original command is close to a multiple of $T$. In such a case, the 


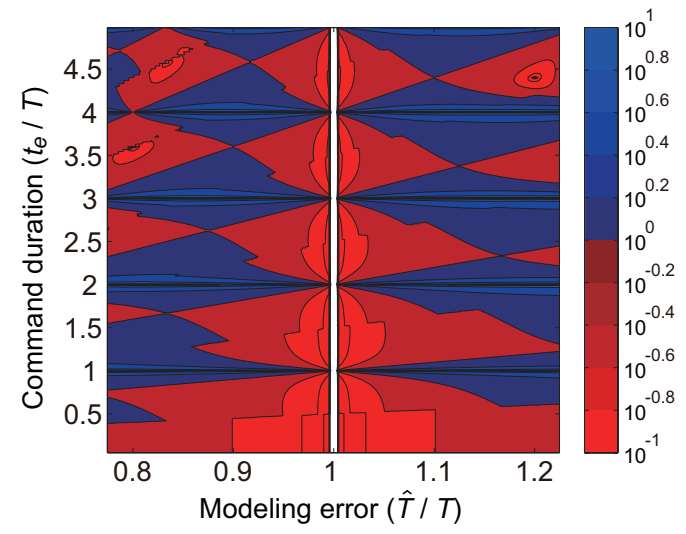

Fig. 10 Comparison of pulse subdivision and normal operation. In red areas, pulse subdivision realizes smaller residual oscillations than the normal operation, whereas in blue areas, the use of pulse subdivision enlarges the oscillations.

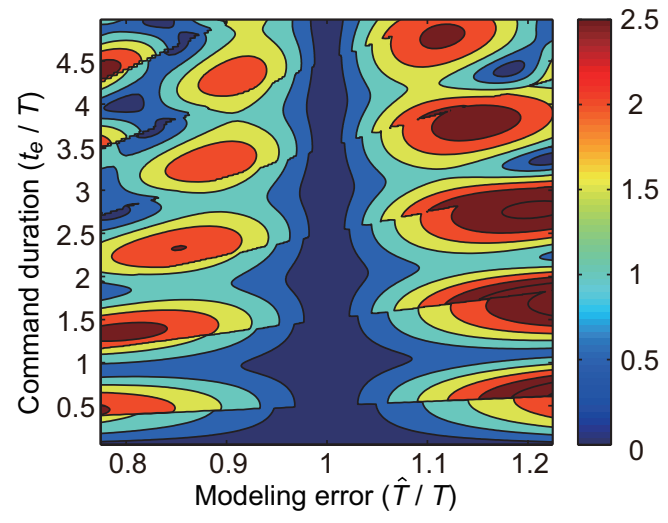

(a) sensitivity of "pulse subdivision + rewind pulse"

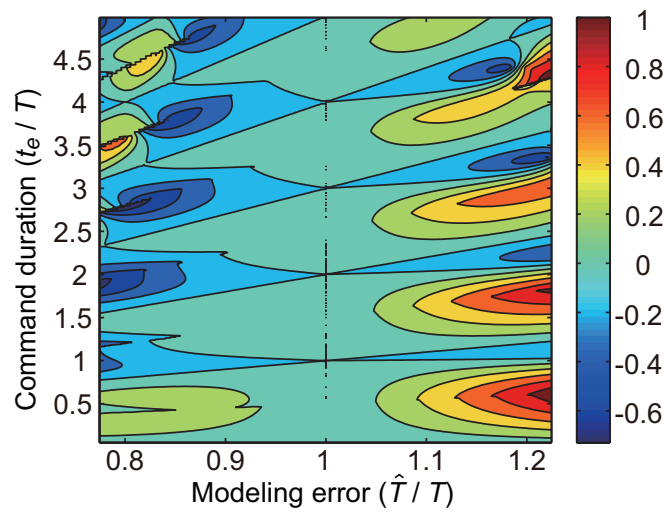

(b) difference from pulse subdivision (Fig. 11(a) - Fig. 8(a))

Fig. 11 Amplitudes of the residual oscillation for pulse subdivision plus rewind pulse. The general tendency is close to that for pulse subdivision alone. This means that pulse subdivision is dominant in determining the residual oscillation.

residual oscillation does not occur with the original command, and no oscillation suppression pulse is needed. However, if there is a modeling error, the algorithm adds an unnecessary suppression pulse that enlarges the residual oscillation.

\subsection{Sensitivity of rewind pulse}

When a rewind pulse is added for canceling over-travel, the impulses can be described as

$$
\left\{\begin{array}{l}
{\left[A_{1}, A_{2}, A_{3}, A_{4}, A_{5}, A_{6}\right]=[1,-1,1,-1,-1,1]} \\
{\left[t_{1}, t_{2}, t_{3}, t_{4}, t_{5}, t_{6}\right]=\left[0, t_{e}, t_{e} / 2+(n+1) \hat{T}_{h} / 2, t_{e}+\hat{T}_{h},(n+1) \hat{T}_{h}, t_{e} / 2+(n+3) \hat{T}_{h} / 2\right]}
\end{array}\right.
$$

for the case of positive suppression pulse. For the other case,

$$
\left\{\begin{array}{l}
{\left[A_{1}, A_{2}, A_{3}, A_{4}, A_{5}, A_{6}\right]=[1,-1,-1,1,1,-1]} \\
{\left[t_{1}, t_{2}, t_{3}, t_{4}, t_{5}, t_{6}\right]=\left[0, t_{e}, t_{e} / 2+(n+1) \hat{T}_{h} / 2, n \hat{T}_{h}, t_{e}+2 \hat{T}_{h}, t_{e} / 2+(n+3) \hat{T}_{h} / 2\right]}
\end{array}\right.
$$

The calculated amplitudes of the residual oscillation is plotted in Fig. 11(a) and the difference against Fig. 8(a) is shown in (b). Although, on average, the oscillation amplitude slightly increases by addition of a rewind pulse, the general tendency does not largely change from the result of pulse subdivision alone. This means that the former part of the algorithm, which is pulse subdivision, is more dominant in determining the residual oscillation.

On the whole, the evaluated methods, which are pulse subdivision and rewind pulse, are found sensitive to the duration of the original command. For a shorter command pulse, the methods can effectively suppress the oscillation even with some modeling errors. However, for a longer command pulse, the oscillation period should be precisely determined for successful oscillation suppression. With modeling errors, the algorithm may even enlarge the oscillation for a long input command pulse. Regarding the other two advantages of the evaluated algorithm, which are no start-up delay and no 
over-travel, the duration of the input command does not matter. With any command length, the algorithm guarantees no start-up delay and no over-travel, in its principle.

\section{Conclusions}

Anti-sway control for flexibly suspended objects has been studied in many literatures. However, its application to fixed-speed cranes has been limited. Although there is a method applicable to fixed-speed cranes, it suffers from start-up delay and over-travel issues. In this work, by exploiting the nature of ZV shaper, a simple anti-sway control algorithm has been implemented and evaluated in terms of its robustness to the modeling error. The implemented method consists of two parts: pulse subdivision and rewind pulse. In the experiments, the effect of these two parts were successfully verified; the pulse-subdivision realized no start-up delay and rewind pulse canceled out the over-travel, without residual oscillations. On the other hand, the method does not suppress the oscillation during transport. Therefore, the method is not applicable to the applications where oscillations during transport is not allowed.

The latter half of the paper analyzed the robustness of the method. The analysis revealed that the method is sensitive to the duration of the operation command. This would be the largest drawback of this simple algorithm, compared to typical input shapers whose performance is not affected by the command duration. The implemented method was found effective for a short operation command. Therefore, it could be applied to cranes with long hoist cables and/or short traveling distance. For longer operation command, one possible solution is to use UM-shaper, instead of pulse subdivision. Since the concept of rewind pulse can be utilized to compensate the over-travel in other algorithms, the use of rewind pulse can suppress over-travel even in UM shaper, although it does not have the advantage of no start-up delay.

Another solution would be a combination with feedback control. The high modeling sensitivity in long operation command comes from the fact that the modeling error accumulates as the command duration becomes long. If a crane is equipped with a sensor to monitor the oscillation period, the error accumulation can be compensated and more robust oscillation suppression would be realized in a simple manner. Our future work will address such feedback compensation.

Finally, the theory described in the paper dealt with linear systems. Any non-linearity that may exist in a real system was ignored. Also in the experiments, we implemented a velocity feedback controller to minimize the effect of friction and other non-linearities. In actual systems, however, frictions and other non-linearities may exist, which can thwart a successful implementation of the proposed method. Therefore, clarifying the range of applicable systems is another important future work.

\section{References}

Alhassan, A., Danapalasingam, K. A., Shehu, M., Abdullahi, A. M., and Shehu, A., Comparing the performance of sway control using ZV input shaper and LQR on gantry cranes, Proceedings of 2015 9th Asia Modelling Symposium (2015), pp. 61-66.

Cutforth, C. F., and Pao, L. Y., Control using equal length shaped commands to reduce vibration, IEEE Transactions on Control Systems Technology, Vol. 11, No. 1 (2003), pp. 62-72.

Gürleyük, S., Optimal unity-magnitude input shaper duration analysis, Arch Appl Mech, Vol. 77, No. 1 (2007), pp. 63-71. Miyata, N., Ukita, T., Nishioka, M., Monzen, T., Toyohara, T., Development of Feedforward Anti-Sway Control for Highly efficient and Safety Crane Operation, Mitsubishi Heavy Industries, Ltd. Technical Review, Vol. 38, No. 2 (2001), pp. 73-77.

Pao, L. Y., and Singhose, W. E., Unity-magnitude input shapers and their relation to time-optimal control, IFAC Proceedings Volumes, 13th IFAC World Congress, Vol. 29, No. 1 (1996), pp. 385 - 390.

Singer, N., Residual Vibration Reduction in Computer Controlled Machines, Ph.D thesis, Dept of Mechanical Engiinering, MIT, AI-TR-1030 (1988).

Singer, N., and P. Seering, W., Preshaping command inputs to reduce system vibration, Journal of Dynamic Systems Measurement and Control, Vol. 112, No. 1 (1990), pp. 76-82.

Singer, N., Singhose, W., and Kriikku, E., An input shaping controller enabling cranes to move without sway, Proceedings of American Nuclear Society 7th Topical Meeting on Robotics and Remote Systems (1997), pp. $225-231$.

Singhose, W. E., Seering, W. P., and Singer, N. C., Shaping inputs to reduce vibration: a vector diagram approach, Proceedings of IEEE International Conference on Robotics and Automation, Vol. 2 (1990), pp. 922-927.

Singhose, W., P. Seering, W., and C Singer, M., Input shaping for vibration reduction with specified insensitivity to 
modeling errors, In Proceedings of the 1996 Japan-USA Symposium on Flexible Automation, Vol. 1 (1996), pp. 307313.

Singhose, W., Command shaping for flexible systems: A review of the first 50 years, International Journal of Precision Engineering and Manufacturing, Vol. 10, No. 4 (2009), pp. 153-168.

Smith, O. J. M., Posicast control of damped oscillatory systems, Proceedings of the IRE, Vol. 45, No. 9 (1957), pp. 12491255.

Sorensen, K., Singhose, W., and Dickerson, S., A controller enabling precise positioning and sway reduction in cranes with on-off actuation, IFAC Proceedings Volumes, 16th IFAC World Congress, Vol. 38, No. 1 (2005), pp. 580 - 585.

Vaughan, J., and Singhose, W., Input shapers for reducing overshoot in human-operated flexible systems, Proceedings of 2009 American Control Conference (2009), pp. 178-183.

Vaughan, J., Jurek, P., and Singhose, W., Reducing overshoot in human-operated flexible systems, Journal of Dynamic Systems, Measurement, and Control, Vol. 133, No. 1 (2011), pp. 011010.1-10.

Wada, M., Mori, Y., Tagawa, Y., Honma, K., Development of Practical Application for Sensorless Crane Control System, Proceedings of 2017 11th Asian Control Conference (2017), pp. 1320-1325.

Yaovaja, K., Chatlatanagulchai, W., and Yaemprasuan, P., Anti-delay closed-loop input shaper to improve manual control of flexible system, Proceedings of 2017 American Control Conference (2017), pp. 4498-4503.

Zhao, Y., Chen, W., Tang, T., and Tomizuka, M., Zero time delay input shaping for smooth settling of industrial robots, Proceedings of 2016 IEEE International Conference on Automation Science and Engineering (CASE) (2016), pp. 620-625.

Zhao, Y., and Tomizuka, M., Modified zero time delay input shaping for industrial robot with flexibility, Proceedings of ASME 2017 Dynamic Systems and Control Conference (2017) p. V003T22A003. 